\title{
Avaliação da variabilidade do estado nutricional e produtividade de café por meio da análise de componentes principais e geoestatística
}

\author{
Samuel de Assis Silva ${ }^{2 *}$ e Julião Soares de Souza Lima ${ }^{3}$
}

\section{RESUMO}

Determinar o estado nutricional das plantas traz diversas contribuições à cafeicultura, principalmente quando esse estado é avaliado, considerando-se a variação espacial existente nos campos de produção. Este experimento objetivou analisar a variabilidade espacial do estado nutricional e da produtividade de café arábica, utilizando técnicas de análise de componentes principais (ACP) e geoestatística. O experimento foi desenvolvido em uma malha de 50 pontos amostrais, montada em uma lavoura de Coffea arabica L., variedade Catuaí. Realizaram-se amostragens foliares para avaliação dos macro e micronutrientes e coletas de frutos para o levantamento da produtividade. Os dados foram submetidos à ACP, para transformação dos valores de nutrientes em novas variáveis e, em seguida, submetidos à geoestatística, para quantificar o grau de dependência espacial dos componentes principais e da produtividade. A aplicação conjunta de ACP e geoestatística permitiu estudar de forma eficiente o estado nutricional das plantas. A lavoura estudada apresenta desequilíbrio nutricional, sendo o excesso de $\mathrm{N}$ e de $\mathrm{Cu}$ limitantes à produtividade da cultura, mesmo em locais com adequada concentração foliar dos demais nutrientes.

Palavras-chave: análise multivariada, nutrição vegetal, análise foliar, Coffea arabica L.

\section{ABSTRACT}

\section{Assessment of the variability of the nutritional status and yield of coffee by principal component analysis and geostatistics}

Evaluation of plant nutritional status brings several contributions to the coffee crop, especially when considering the spatial variation in the production fields. This experiment aimed to analyze the spatial variability in the nutritional status and yield of arabic coffee, using the principal component analysis (PCA) and geostatistics. The experiment was conducted in a grid of 50 sampling points in a field of Coffea arabica L., cv. Catuaí. Leaf samples were taken for macro and micronutrient analyses, and fruit were collected for yield evaluation. Data were subjected to PCA for transformation of nutrient values into new variables and then submitted to geostatistics to quantify the degree of spatial dependence of the main components and yield. The combined use of PCA and geostatistics allowed the efficient study of the nutritional status of the plants. The crop showed nutritional imbalance, with excess $\mathrm{N}$ and $\mathrm{Cu}$ limiting yield even in areas with adequate leaf concentration of the other nutrients.

Key words: multvariate analysis, vegetal nutrition, foliar analysis, Coffee arabica L.

\footnotetext{
Recebido para publicação em 21/03/2011 e aprovado em 19/12/2011

${ }^{1}$ Parte da dissertação de Mestrado do primeiro autor.

${ }^{2}$ Engenheiro-Agrônomo, Mestre. Departamento de Ciências Agrárias e Ambientais, Universidade Estadual de Santa Cruz, Rodovia Ilhéus/Itabuna, Km 16, 45662-900, Ilhéus, Bahia, Brasil.samuel-assis@hotmail.com *Autor para correspondência.

${ }^{3}$ Engenheiro Agrícola, Doutor. Departamento de Engenharia Rural, Universidade Federal do Espírito Santo, Alto Universitário, 20500-000, Alegre, Espírito Santo, Brasil. limajss@yahoo.com.br
} 


\section{INTRODUÇÃO}

A cafeicultura brasileira tem passado por diversas modificações, na busca de um sistema de cultivo mais autossustentável (Petek et al., 2006). Este fato torna fundamental o estudo e o desenvolvimento de novas técnicas de manejo da cultura, buscando a redução de perdas decorrentes de manejos inadequados e consequentes quebras de produtividade, ou, mesmo, o alcance de potenciais produtivos além daqueles previstos para as variedades atualmente cultivadas.

A adoção de manejos adequados, que considerem tanto a fertilidade do solo, como o estado nutricional das plantas, deve ser considerada (Silva et al., 2011). A grande exportação de nutrientes do solo, pela cultura do café, e o elevado preço dos adubos têm feito com que o monitoramento nutricional de cafezais, mediante análise química das folhas, transforme-se em prática essencial para recomendação de adubações mais equilibradas e economicamente mais ajustadas (Bataglia et al., 2004).

A análise química foliar vem sendo usada para detectar respostas de cafeeiros aos vários tipos de manejo, possibilitando interpretar, de maneira mais eficiente, as relações entre nutrientes na planta, e interferir no sistema, elevando as possibilidades de melhores resultados produtivos (Farnezi et al., 2010). Segundo Corrêa et al. (2001), a maior vantagem no estudo da nutrição das plantas está no fato de se considerar a própria planta como extrator dos nutrientes no solo e permitir uma avaliação direta de seu estado nutricional e, desse modo, avaliar as concentrações e as relações entre nutrientes.

Para analisar o estado nutricional das plantas, é necessária a avaliação quantitativa dos diversos elementos químicos classificados como nutrientes. A interpretação dos resultados por métodos estatísticos univariados muitas vezes é falha. Uma opção de análise dos resultados é o uso de componentes principais, que permite a redução dimensional do problema, além de aumentar a eficiência de sua interpretação e a escolha da solução a ser adotada (Hair et al., 2005).

A análise de componentes principais é realizada, frequentemente, com base na matriz de correlação dos dados, de forma a identificar novas variáveis que expliquem a maior parte da variabilidade. Em uma matriz original de dados, cada sítio tem um valor para cada atributo. Na análise de componentes principais, cada sítio tem um valor para cada componente (Kent \& Coker, 1992).

A técnica de componentes principais, associada à geoestatística, tem permitido abordagens eficientes em ciências agrárias, principalmente por considerar, simultaneamente, a variação espacial de um número elevado de variáveis que influenciam os sistemas de produção agrícola (Silva et al., 2010). Estudar essa variação espacial é muito importante, pois o tratamento uniforme de áreas agrícolas pode resultar em locais com superdoses de insumos ou em áreas mal corrigidas, o que seria impactante do ponto de vista econômico e ecológico (Viscarra-Rossel \& McBratney, 1998).

Diante do exposto, este trabalho foi conduzido com o objetivo de analisar a variabilidade espacial do estado nutricional e da produtividade de plantas de café arábica, variedade Catuaí, utilizando, de forma conjunta, as técnicas de análise de componentes principais (ACP) e a geoestatística.

\section{MATERIAL E MÉTODOS}

O estudo foi desenvolvido em uma área experimental situada a $20^{\circ} 45^{\prime} 45,4^{\prime}$ de latitude $S$ e $41^{\circ} 32^{\prime}$ ' 9,75' de longitude W e altitude média de 827 metros, localizada na Fazenda Jaguaraí, no município de Reduto, Zona da Mata de Minas Gerais.

A área, com aproximadamente 0,8 ha, vem sendo cultivada há 5 anos com Coffea arabica L., variedade Catuaí Vermelho 44, no espaçamento de 2,5 x 0,6 m, sendo o solo de toda a área classificado como Latossolo VermelhoAmarelo húmico com horizonte A bastante espesso e rico em matéria orgânica, conforme Embrapa (1999).

Construiu-se uma malha de amostragem contendo 50 pontos amostrais, onde foram realizadas as amostragens. Os pontos foram marcados com estacas de madeira e o levantamento topográfico foi realizado por meio de uma estação total. O sistema de coordenadas utilizado foi o de coordenadas topográficas locais.

Cada ponto amostral, com uma área aproximada de $6 \mathrm{~m}^{2}$, foi composto de três plantas. Para análise foliar (estado nutricional), foram coletadas nos quatro pontos cardeais de cada uma das plantas que compõem a célula, na porção mediana, folhas do terceiro e quarto pares dos ramos produtivos, sendo determinados os teores de macronutrientes ( $\mathrm{N}, \mathrm{P}, \mathrm{K}, \mathrm{Ca}, \mathrm{Mg}$ e $\mathrm{S}$ ) e micronutrientes ( $\mathrm{Zn}, \mathrm{Cu}, \mathrm{Fe}, \mathrm{Mn}$ e B). Essa caracterização do estado nutricional foi realizada no mês de dezembro de 2007, estando as plantas no estádio de primeira expansão rápida.

A produção foi avaliada em julho de 2008, sendo a colheita em cada ponto feita por derriça manual. Em seguida, procedeu-se à pesagem do café cereja colhido de cada ponto (massa de café úmido) e uma amostra de 1,0 $\mathrm{kg}$ foi colocada em estufa a $70^{\circ} \mathrm{C}$, até atingir $\pm 12 \%$ de umidade. Com o café já seco, efetuaram-se o beneficiamento e o cálculo de rendimento, convertendo-se os valores em produção de café beneficiado por hectare, de acordo com Tomaz et al. (2005).

Os valores encontrados foram submetidos à uma análise exploratória para verificar a presença de valores dis- 
crepantes (outliers) e sua influência sobre as medidas de posição e dispersão. Os dados foram analisados por meio das medidas de posição (média e mediana), dispersão (valores máximos, mínimos, desvio padrão, variância e coeficiente de variação) e forma da dispersão (coeficientes de assimetria e curtose). A normalidade foi testada pelo teste Shapiro-Wilk's ( $\mathrm{p}<0,05)$.

A análise de componentes principais (ACP) foi realizada com base na diagonalização de sua matriz de correlação simétrica, após análise da variância populacional, conforme default do software. Essa análise foi realizada de forma a identificar novas variáveis que expliquem a maior parte da variabilidade. A seleção do número de componentes principais foi baseada no critério de análise da qualidade de aproximação da matriz de correlações, denominado método de Kaiser ou Método da Raiz Latente, utilizando-se os componentes associados a autovalores superiores a 1 (Hair et al., 2005; Mingoti, 2007; Silva et al., 2010) e na correlação dos componentes com os atributos químicos do solo. As análises estatísticas uni e multivariadas foram realizadas, utilizando-se o software Statistica, versão 7.0.

A geoestatística foi utilizada para verificar a existência e, neste caso, quantificar o grau de dependência espacial dos dados gerados, por meio da aplicação da ACP. A análise foi feita a partir do ajuste de funções teóricas aos modelos de variogramas experimentais, com base na pressuposição de estacionaridade da hipótese intrínseca e conforme a equação:

$$
\gamma^{*}(h)=\frac{1}{2 N(h)} \sum_{i=1}^{N(h)}\left[z\left(x_{i}\right)-z\left(x_{i}+h\right)\right]^{2}
$$

em que: N(h) é o número de pares experimentais de observações $\mathrm{Z}(\mathrm{xi}), \mathrm{Z}(\mathrm{xi}+\mathrm{h})$, separados por um vetor $\mathrm{h}$ (Vieira, 2000).

No ajuste dos modelos teóricos aos variogramas experimentais, foram testados os modelos esférico, exponencial, gaussiano e linear. Determinaram-se, com o software $\mathrm{GS}+$, os coeficientes efeito pepita $\left(\mathrm{C}_{0}\right)$, patamar $\left(\mathrm{C}_{0}+\mathrm{C}\right)$, variância estrutural $(\mathrm{C})$ e alcance (a). O critério para adoção dos modelos foi o maior valor de $\mathrm{R}^{2}$ (coeficiente de determinação), a menor SQR (soma de quadrado dos resíduos) e o maior valor do coeficiente de correlação obtido pelo método de validação cruzada.

Para análise do índice de dependência espacial (IDE), foram utilizados a relação $\mathrm{C} 0 /(\mathrm{C} 0+\mathrm{C})$ e os intervalos propostos por Cambardela et al. (1994), que consideraram a dependência espacial fraca (IDE > 75\%), moderada (25\% $\leq \mathrm{IDE} \leq 75 \%)$ e forte (IDE < 25\%).

Comprovada a dependência espacial, utilizou-se o método de interpolação de krigagem ordinária, para estimar valores em locais não medidos, conforme Vieira (2000).

\section{RESULTADOS E DISCUSSÃO}

Os resultados obtidos pela análise estatística descritiva, após a retirada dos dados candidatos a valores discrepantes (outiliers), encontram-se na Tabela 1. Com exceção de $\mathrm{Fe}, \mathrm{Mn}$ e $\mathrm{Cu}$, todos os nutrientes apresentaram valores de medidas de tendência central (média e mediana) bem próximos. Em relação aos nutrientes para os quais essas medidas foram diferentes, os valores de assimetria apresentaram-se distantes de zero, levando a um afastamento da distribuição normal. Nesse caso, os valores de assimetria foram positivos, demonstrando tendência de concentração dos dados à direita, com valores de média superiores à mediana. Os demais nutrientes apresentaram coeficientes de assimetria próximos de zero, sugerindo um ajuste à distribuição normal, o que não se confirmou apenas para $\mathrm{Mg}$, Zn, Cu e Mn, conforme teste de ShapiroWilk's a 5\% de probabilidade. O afastamento desses nutrientes da normalidade deve-se à associação entre altos valores de assimetria e curtose, indicando tanto o alongamento da cauda, à direita da curva de distribuição normal, quanto o achatamento da mesma.

Analisando-se o coeficiente de variação (CV) (Tabela 1), observa-se que, segundo a classificação proposta por Warrick \& Nielsen (1980), (CV < 12\% - baixos; $12 \%<\mathrm{CV}<$ $60 \%$ - médios; e CV > 60\% - altos), com exceção do N (baixo), os demais nutrientes foliares encontram-se no intervalo de 12 a $60 \%$, sendo, portanto, considerados de média variação.

A lavoura apresenta-se em desequilíbrio nutricional, uma vez que os teores foliares médios dos nutrientes são classificados, segundo Ribeiro et al. (1999), como baixos para $\mathrm{K}, \mathrm{Mg}$, S e Zn, elevados para $\mathrm{N}$ e $\mathrm{Cu}$ e adequados para P, Ca, Fe, Mn e B. Martinez et al. (2008) afirmam que os nutrientes $\mathrm{K}, \mathrm{Mg}$, S e Zn são de extrema importância para o crescimento vegetativo e desenvolvimento dos frutos da planta de café, sendo que o desequilíbrio desses elementos pode acarretar significativas perdas na produtividade da cultura.

$\mathrm{Na}$ análise de componentes principais, foram extraídos oito componentes principais que, de forma acumulada, explicam $91,75 \%$ da variabilidade total dos dados, conforme Tabela 2. Como a contribuição dos demais componentes foi mínima, eles não foram considerados na análise.

Conforme o critério de seleção já discutido, as análises foram realizadas, utilizando os dois primeiros componentes principais. $\mathrm{O}$ primeiro componente apresenta isoladamente $26,31 \%$ da variabilidade total, enquanto, o segundo, 23,77\%. A partir do terceiro componente, a contribuição para explicar a variância dos dados acresce-se minimamente, sendo que estes se correlacionam com um número reduzido de nutrientes (Tabela 3), o que motivou a sua exclusão de análises posteriores. 
Tabela 1. Estatística descritiva e distribuição de frequência dos teores foliares de nutrientes

\begin{tabular}{|c|c|c|c|c|c|c|c|c|c|}
\hline \multirow{2}{*}{ Nutrientes } & \multicolumn{9}{|c|}{ Estatística } \\
\hline & Média & Mediana & Mínimo & Máximo & CV\% & $\mathbf{s}$ & $\mathrm{C}_{\mathrm{s}}$ & $\mathrm{C}_{\mathrm{k}}$ & $\mathbf{w}$ \\
\hline$N$ & 3,23 & 3,24 & 2,57 & 3,94 & 10,43 & 0,34 & 0,10 & $-0,28$ & $\mathrm{~ns}$ \\
\hline $\mathrm{P}$ & 0,14 & 0,15 & 0,08 & 0,23 & 23,59 & 0,03 & 0,17 & $-0,17$ & $\mathrm{~ns}$ \\
\hline K & 1,22 & 1,20 & 0,85 & 1,65 & 14,14 & 0,17 & 0,34 & 0,13 & $\mathrm{~ns}$ \\
\hline $\mathrm{Ca}$ & 1,04 & 1,02 & 0,69 & 1,46 & 17,00 & 0,18 & 0,10 & $-0,22$ & ns \\
\hline $\mathrm{Mg}$ & 0,24 & 0,22 & 0,17 & 0,33 & 22,10 & 0,05 & 0,09 & $-1,14$ & * \\
\hline S & 0,08 & 0,08 & 0,05 & 0,11 & 14,97 & 0,01 & 0,27 & 0,00 & $\mathrm{~ns}$ \\
\hline $\mathrm{Zn}$ & 7,75 & 7,85 & 4,10 & 10,60 & 22,74 & 1,76 & $-0,25$ & $-0,67$ & * \\
\hline $\mathrm{Fe}$ & 89,24 & 84,85 & 51,60 & 149,60 & 26,31 & 23,48 & 0,70 & $-0,14$ & $\mathrm{~ns}$ \\
\hline $\mathrm{Mn}$ & 133,04 & 126,00 & 84,90 & 215,50 & 26,07 & 34,68 & 0,66 & $-0,67$ & * \\
\hline $\mathrm{Cu}$ & 34,25 & 32,45 & 10,95 & 72,45 & 47,19 & 16,16 & 0,56 & $-0,63$ & * \\
\hline $\mathrm{B}$ & 52,91 & 52,30 & 38,70 & 67,10 & 11,94 & 6,32 & 0,16 & $-0,16$ & $\mathrm{~ns}$ \\
\hline
\end{tabular}

$\mathrm{s}=$ desvio padrão; $\mathrm{CV}(\%)=$ coeficiente de variação; $\mathrm{C}_{\mathrm{s}}=$ assimetria; $\mathrm{C}_{\mathrm{k}}=$ curtose; $\mathrm{N}, \mathrm{P}, \mathrm{K}, \mathrm{Ca}, \mathrm{Mg} \mathrm{e} \mathrm{S} \mathrm{em} \mathrm{dag} \mathrm{kg-1,} \mathrm{Zn}, \mathrm{Fe}, \mathrm{Mn}, \mathrm{Cu}, \mathrm{B}$ em mg.kg-1; * distribuição não normal pelo teste de Shapiro-Wilk's a 5\% de probabilidade; e ns distribuição normal pelo teste de Shapiro-Wilk's a $5 \%$ de probabilidade.

O primeiro componente correlaciona-se com sete dos onze nutrientes analisados no tecido foliar. $\operatorname{Com~N,~P,~Zn~e~}$ $\mathrm{Cu}$ essa correlação é negativa, enquanto, com os demais nutrientes ( $\mathrm{Ca}, \mathrm{Mg}$ e Fe), as correlações com esse componente foram positivas. Assim sendo, quando os níveis de $\mathrm{Ca}, \mathrm{Mg}$ e Fe elevam-se, ocorre uma redução nos valores de $\mathrm{N}, \mathrm{P}, \mathrm{Zn}$ e $\mathrm{Cu}$, sendo o inverso também verdadeiro.

Com exceção do $\mathrm{Ke} \mathrm{S}$, esse primeiro componente apresenta correlação elevada com todos os macronutrientes foliares (67\% dos nutrientes). Desta forma, representa os nutrientes englobados nessa classe, correspondendo ao estado nutricional das plantas. Esse componente apresenta também correlação com três dos cinco micronutrientes (60\% dos nutrientes), entretanto não pode representar essa classe uma vez que existe, dentre os componentes selecionados, um que se correlacionou com todos os nutrientes da mesma. De acordo com Hair et al. (2005), na interpretação dos componentes principais a contribuição das variáveis deve ser analisada de forma individual apenas quando essas forem independentes para a expressão da característica em estudo, quando a ação dessas for conjunta ou as mesmas formarem um grupo de variáveis, a interpretação dos componentes deve se basear na contribuição do grupo. Mingoti (2007) afirma que, para a atribuição de uma componente a um dado fenômeno, devese priorizar aquela que se correlaciona com o maior número de variáveis que caracterizam tal fenômeno.

O segundo componente se correlaciona com todos os micronutrientes foliares, desta forma, esse representa essa classe de nutrientes. Além desses, esse componente se correlaciona com os valores de $\mathrm{S}$. As correlações foram negativas para $\mathrm{Zn}$, Mn e B, enquanto que os demais apresentaram correlações positivas com esse componente. Diante disso, locais onde os valores de $\mathrm{Zn}, \mathrm{Mn}$ e B são baixos, há elevada concentração de $\mathrm{S}, \mathrm{Fe}$ e $\mathrm{Cu}$.
A interação entre micronutrientes tem sido relatada por diversos autores (Fageria, 2001; Kaya \& Higgs, 2001; Marschner, 1995). O excesso de zinco, por exemplo, afeta a absorção e a translocação de ferro, pela inibição competitiva entre os cátions, possivelmente pela semelhança de raio iônico entre eles (Marschner, 1995), o que representa prejuízos ao desenvolvimento das plantas e, consequentemente, redução da produção.

A análise geoestatística, com o intuito de mapear o estado nutricional e a produtividade das plantas, foi realizada utilizando-se os valores do primeiro e do segundo componentes principais, e os valores de produtividade, sendo os resultados apresentados na Tabela 4.

Os componentes principais apresentaram dependência espacial, indicando que a distribuição dos nutrientes foliares nas áreas não é aleatória, mas sofre variação em função da distância entre as amostras.

A semivariância estabiliza-se (atinge o patamar) com alcances de 73,0 e 56,0 m, para o primeiro e o segundo componente, respectivamente, indicando diferença no grau de continuidade espacial dos nutrientes. Silva et al. (2010), utilizando análise de componentes principais e

Tabela 2. Resumo dos componentes principais da análise multivariada dos teores foliares de nutrientes

\begin{tabular}{lccc}
\hline $\begin{array}{l}\text { Componente } \\
\text { Número }\end{array}$ & Autovalor & $\begin{array}{c}\text { Percentagem } \\
\text { daVariância }\end{array}$ & $\begin{array}{c}\text { Percentagem } \\
\text { acumulada }\end{array}$ \\
\hline 1 & 2,89 & 26,31 & 26,31 \\
2 & 1,51 & 23,77 & 50,08 \\
3 & 1,42 & 10,93 & 61,01 \\
4 & 1,20 & 10,04 & 71,05 \\
5 & 0,97 & 7,84 & 78,88 \\
6 & 0,83 & 6,51 & 85,39 \\
7 & 0,65 & 3,90 & 89,29 \\
8 & 0,49 & 2,47 & 91,75 \\
\hline
\end{tabular}


geoestatística para a avaliação da fertilidade de um Latossolo, também encontraram valor maior de alcance para o primeiro componente. Esses autores atribuíram esse fenômeno à percentagem de variância contida nos componentes, ou seja, quanto maior essa, maior a continuidade espacial do componente.

Segundo Zanão Júnior et al. (2007), a variabilidade espacial dos nutrientes pode não ser igual entre eles, ou seja, alguns nutrientes necessitam de números maiores de amostras que outros para que se possa conhecer o seu comportamento em relação a uma determinada área.

O segundo componente, que representou os micronutrientes foliares, apresenta menor continuidade espacial que o primeiro componente e, consequentemente, maior variabilidade espacial. Sendo que, para tal, amostragens capazes de representar a distribuição dos elementos que a compõem devem ser feitas em distâncias menores, o que implica maiores quantidades de amostras por unidade de área.

O primeiro componente principal apresenta forte dependência espacial, analisada pelo índice de dependência espacial (IDE), de acordo com classificação sugerida por Cambardella et al. (1994). Segundo essa classificação, o segundo componente apresentou moderada dependência espacial, a qual é resultado do elevado valor de $\mathrm{C}_{0}$, mostrando que uma proporção reduzida do patamar está ocupada pela variância estrutural (C), o que pode comprometer as estimativas de valores em locais não medidos (Vieira, 2000).
Ao variograma da produtividade, ajustou-se o modelo esférico com alcances de $14 \mathrm{~m}$ para a produtividade. $\mathrm{O}$ índice de dependência espacial foi elevado para a produtividade, de acordo com a classificação de Cambardella et al. (1994). Quanto ao valor de $\mathrm{C}_{0}$, a produtividade apresentou baixo valor proporcional, indicando que a variabilidade espacial não é creditada a valores inespecíficos e que estimativas por krigagem ordinária têm precisão elevada.

Com base nos modelos de semivariância e levando-se em consideração os parâmetros ajustados, os dados dos componentes principais e da produtividade foram interpolados por meio da krigagem ordinária (Figura 1).

Pelo mapa do primeiro componente, o qual representa os macronutrientes foliares, foi possível perceber que a maior disponibilidade desses nutrientes ocorre na porção inferior da área, com decréscimo gradativo quando se elevam as cotas. A maior proporção das plantas da área apresenta valores de macronutrientes aquém da necessidade para um bom desenvolvimento e produtividade. Esses valores são impulsionados pela escassez de $\mathrm{K}, \mathrm{Mg}$ e $\mathrm{S}$, conforme discutido anteriormente.

Para os micronutrientes, representados pelo segundo componente, a porção inferior da área apresentou, em semelhança com os macronutrientes, maior proporção de plantas nutricionalmente equilibradas, com plantas, cujo estado nutricional é deficiente, situadas na porção superior direita da área.

Tabela 3. Correlação entre variáveis originais e componentes principais dos teores foliares de nutrientes

\begin{tabular}{lcrrrrrrr}
\hline \multirow{2}{*}{ Atributo } & \multicolumn{7}{c}{ Componente } \\
\cline { 2 - 10 } & $\mathbf{1}$ & $\mathbf{2}$ & $\mathbf{3}$ & $\mathbf{4}$ & $\mathbf{5}$ & $\mathbf{6}$ & $\mathbf{7}$ & $\mathbf{8}$ \\
\hline $\mathrm{N}$ & $\mathbf{0 , 4 3 5}$ & 0,321 & $\mathbf{- 0 , 6 7 1}$ & $-0,188$ & $-0,046$ & 0,121 & $-0,312$ & $-0,196$ \\
$\mathrm{P}$ & $-0,609$ & 0,383 & 0,093 & $-0,337$ & $-0,004$ & 0,236 & 0,311 & 0,095 \\
$\mathrm{~K}$ & 0,227 & 0,375 & $-0,190$ & $\mathbf{- 0 , 4 4 6}$ & $\mathbf{0 , 6 9 9}$ & $-0,021$ & $-0,061$ & 0,142 \\
$\mathrm{Ca}$ & 0,767 & 0,350 & $-0,055$ & 0,177 & $-0,007$ & $-0,084$ & 0,342 & 0,202 \\
$\mathrm{Mg}$ & $\mathbf{0 , 4 1 4}$ & 0,293 & $\mathbf{- 0 , 6 3 5}$ & $-0,093$ & $-0,366$ & $-0,284$ & 0,226 & $-0,039$ \\
$\mathrm{~S}$ & 0,242 & $\mathbf{0 , 5 8 7}$ & 0,090 & 0,313 & $-0,277$ & 0,565 & $-0,200$ & 0,121 \\
$\mathrm{Zn}$ & $\mathbf{0 , 5 0 5}$ & $\mathbf{- 0 , 4 4 4}$ & $-0,014$ & $\mathbf{0 , 4 9 9}$ & 0,092 & $-0,371$ & 0,005 & $-0,215$ \\
$\mathrm{Fe}$ & $\mathbf{0 , 6 2 5}$ & $\mathbf{0 , 4 5 1}$ & 0,310 & 0,242 & 0,302 & $-0,100$ & $-0,199$ & $-0,191$ \\
$\mathrm{Mn}$ & 0,050 & $\mathbf{- 0 , 3 9 9}$ & $\mathbf{- 0 , 5 9 9}$ & $\mathbf{0 , 4 8 9}$ & 0,231 & 0,082 & $-0,125$ & 0,305 \\
$\mathrm{Cu}$ & $\mathbf{0 , 6 2 7}$ & $\mathbf{0 , 4 3 8}$ & $-0,099$ & $\mathbf{0 , 4 1 1}$ & 0,315 & 0,214 & 0,382 & $-0,075$ \\
$\mathrm{~B}$ & 0,251 & $\mathbf{- 0 , 4 3 0}$ & $-0,218$ & $-0,038$ & 0,133 & 0,386 & 0,205 & $\mathbf{- 0 , 4 3 0}$ \\
\hline
\end{tabular}

* negrito é significativo a $5 \%$ de probabilidade.

Tabela 4. Parâmetros dos variogramas ajustados para os dois componentes principais

\begin{tabular}{lcccccccc}
\hline \multirow{2}{*}{ Variável } & \multicolumn{9}{c}{ Modelos e Parâmetros } \\
\cline { 2 - 11 } & Modelo & $\mathbf{C}_{\mathbf{0}}$ & $\mathbf{C}_{\mathbf{0}}+\mathbf{C}$ & $\mathbf{a}$ & $\mathbf{R}^{\mathbf{2}}$ & $\mathbf{I D E}$ & $\mathbf{R}^{\mathbf{2}}(\mathbf{V C})$ & $\mathbf{p}$-valor \\
\hline Componente 1 & Esférico & 0,31 & 4,0 & 73 & 88 & 08 & 32,60 & 0,00 \\
Componente 2 & Esférico & 0,35 & 0,76 & 56 & 79 & 46 & 36,30 & 0,00 \\
Produtividade & Esférico & 0,10 & 4,00 & 14 & 72 & 02 & 32,70 & 0,00 \\
\hline
\end{tabular}

$\mathrm{C}_{0}$ - efeito pepita; $\mathrm{C}_{0}+\mathrm{C}$ - patamar; IDE - índice de dependência espacial $\left(\mathrm{C} / \mathrm{C}_{0}+\mathrm{C}\right)$; a - alcance; e $\mathrm{R}^{2}$ - coeficiente de determinação do modelo do variograma. 

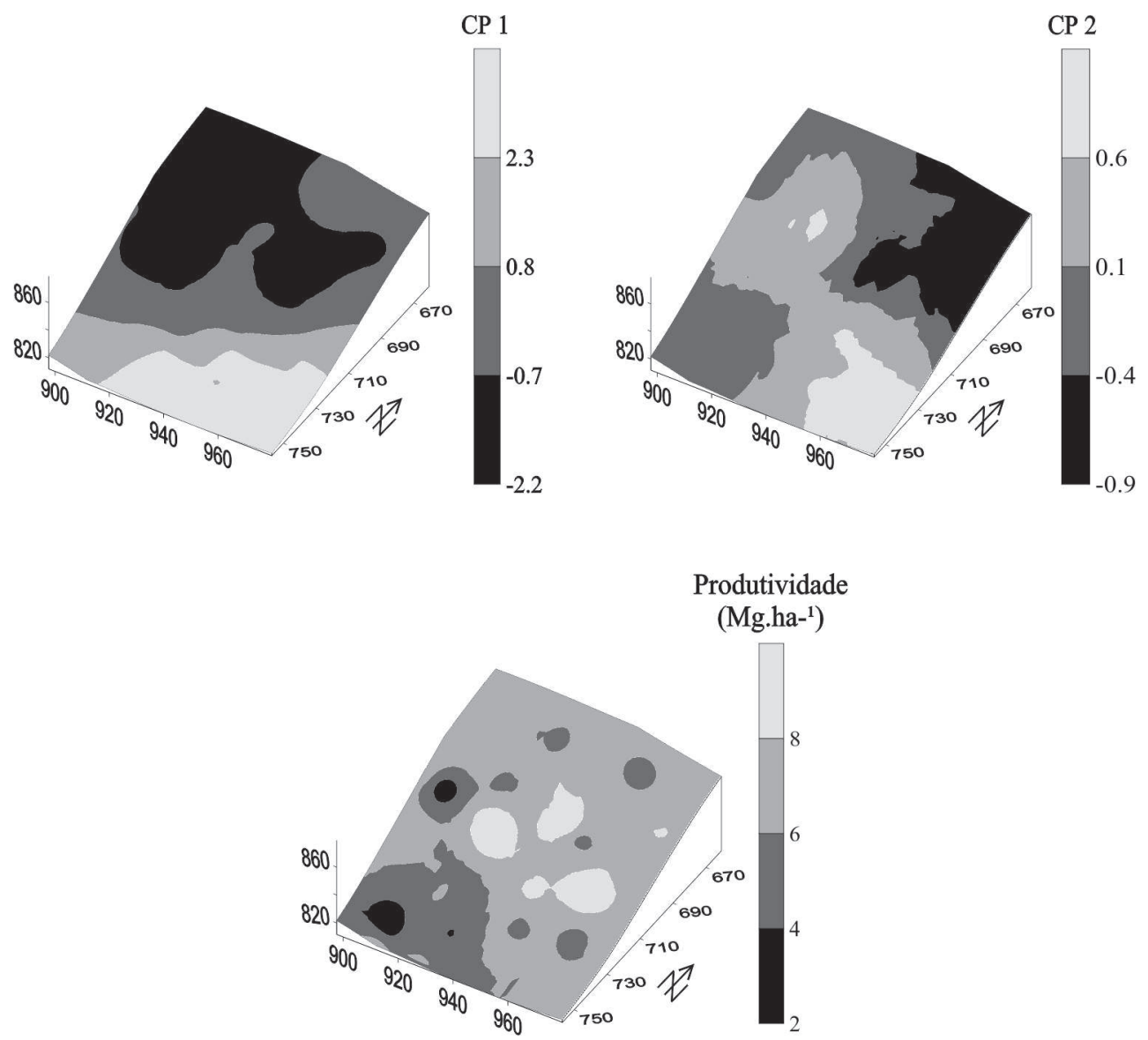

Figura 1. Mapas dos dois primeiros componentes principais (CP1 - macronutrientes e CP2 - micronutrientes) e da produtividade da lavoura de Catuaí avaliada.

Por meio da espacialização da produtividade, é possível perceber que a maior proporção da área em estudo apresentou valores entre 6 e $8 \mathrm{Mg} \mathrm{ha}^{-1}$,o que representa em torno de 100 a $130 \mathrm{sc} \mathrm{ha}^{-1}$. Esses valores de produtividade estão acima dos valores observados em média para café arábica e se justificam pelo espaçamento adensado das plantas.

As menores produtividades são observadas na porção inferior esquerda da área, ocorrendo em locais de maiores disponibilidades de nutrientes. Tal fenômeno pode ser atribuído, principalmente, aos nutrientes $\mathrm{Ne} \mathrm{Cu}$, os quais, segundo classificação de Ribeiro et al. (1999), apresentam-se em nível elevado no solo, inibindo a produtividade da cultura; logo, em locais onde essa concentração é menor, espera-se obter uma maior produtividade.

\section{CONCLUSÕES}

O método de análise multivariada, baseada nos componentes principais forneceu componentes interpretáveis para os teores foliares de nutrientes, sendo o primeiro (teores de N, P, Ca, Mg, Zn, Fe e Cu) e o segundo (teores de $\mathrm{S}, \mathrm{B}, \mathrm{Cu}, \mathrm{Fe}, \mathrm{Mn}$ e $\mathrm{Zn}$ ) capazes de caracterizar a maior proporção da variabilidade dos dados originais.
A aplicação conjunta de PCA e geoestatística permitiu estudar de forma eficiente o estado nutricional das plantas de café arábica.

Há um desequilíbrio nutricional na lavoura de café Catuaí avaliada, expressado por valores deficitários de alguns nutrientes, enquanto outros encontram-se em excesso no tecido vegetal.

$\mathrm{O}$ excesso dos nutrientes $\mathrm{N}$ e $\mathrm{Cu}$ limitou a produtividade da cultura, mesmo em locais com adequada concentração foliar dos demais nutrientes.

\section{AGRADECIMENTOS}

À CAPES, pelo auxílio por meio da concessão de bolsa de mestrado.

\section{REFERÊNCIAS BIBLIOGRÁFICAS}

Bataglia O C, Quaggio JA, Santos WR, Abreu MF (2004) Diagnose nutricional do cafeeiro pelo DRIS variando-se a constante de sensibilidade dos nutrientes de acordo com a intensidade e freqüência de resposta na produção. Bragantia, 2:253-263.

Cambardella CA, Moorman TB, Novak JM, Parkin TB, Karlen DL, Turco RF \& Konopka AE (1994). Field-scale variability of soil properties in central Iowa soils. Soil Science Society American Journal, 58:1501-1511. 
Corrêa JB, Reis Júnior R dos A, Carvalho JG, Guimãraes PTG (2001). Avaliação da fertilidade do solo e do estado nutriciona de cafeeiros do Sul de Minas Gerais. Ciência e Agrotecnologia, 25:1279-1286

Empresa Brasileira de Pesquisa Agropecuária - EMBRAPA (1999). Centro Nacional de Pesquisa de Solos (Rio de Janeiro, RJ). Sistema brasileiro de classificação de solos. Brasília, Produção de Informação, 306p.

Fageria VD (2001). Nutrient interactions in crop plants. Journal of Plant Nutrition, 24:1269-1290.

Farnezi MMM, Silva EB, Guimaraes PTE, Pinto NAVD (2010). Levantamento da qualidade da bebida do café e avaliação do estado nutricional dos cafeeiros do Alto Jequitinhonha, Minas Gerais, através do DRIS. Ciência e Agrotecnologia, 34: 11911198.

Hair JR, Anderson RE, Tatham RL \& Black WC (2005). Análise multivariada de dados. Porto Alegre: Buckman, 593p.

Kaya C \& Higgs D (2001). Inter-relationships between zinc nutrition, growth parameters and nutrient physiology in a hydroponically grown tomato cultivar. Journal of Plant Nutrition, 24:1491-1503.

Kent M \& Coker P (1992). Vegetation description and analysis. Baffins Lane, John Wiley \& Sons, 363p.

Marschner H (1995). Mineral nutrition of higher plants. 2.ed. New York: Academic Press, 889p.

Martinez HEP, Neves YP, Zabini AV, Clemente JM \& Pedrosa AW (2008). Diagnose foliar em cafeeiros. In: Tomaz MA, Amaral JFT, Jesus Júnior WC \& Pezzopane JRM. (Eds.). Seminário para a sustentabilidade da cafeicultura. Alegre/ES, UFES. 342p.

Mingoti SA (2007). Análise de dados através de métodos de estatística multivariada. Belo Horizonte. Editora UFMG, $1^{\text {a }}$ reimpressão, 295p.
Petek MR, Sera T, Sera GH, Fonseca ICB \& Ito DS (2006). Seleção de progênies de Coffea arabica com resistência simultânea à mancha aureolada e à ferrugem alaranjada. Bragantia, 65:6573

Ribeiro AC, Guimarães PTG \& Alvarez VHV (1999). (Ed). Recomendações para uso de corretivos e fertilizantes em Minas Gerais. 5 ${ }^{\text {a }}$ Aproximação. Viçosa, MG: Comissão de Fertilidade do Solo do Estado de Minas Gerais - CFSEMG, 359p.

Silva AS, Lima JSS, Xavier AC \& Teixeira MM (2010). Variabilidade espacial de atributos químicos de um Latossolo vermelhoamarelo húmico cultivado com café. Revista Brasileira de Ciência do Solo, 34:15-22.

Silva SA, Lima JSS, Queiroz DM (2011). Spatial variability in nutritional status of arabic Coffee based on dris index. Revista Ceres, 58:256-261.

Tomaz MA, Sakiyama NS, Martinez HEP, Cruz CD, Pereira AA \& Freitas RS (2005). Porta-enxertos afetando o desenvolvimento de plantas de Coffea arabica L.. Ciência Rural, 35:570-575.

Vieira SR (2000). Geoestatística em estudos de variabilidade espacial do solo. In: NOVAIS, R.F.; ALVAREZ V., V.H.; SCHAEFER, C.E.G.R. (Eds.). Tópicos em ciência do solo. Viçosa, MG: Sociedade Brasileira de Ciência do Solo, 1:1-54

Viscarra-Rossel RA \& McBratney AB (1998). Laboratory evaluation of a proximal sensing technique for simultaneous measurements of soil clay and water content. Goederma, 85:1939.

Warrick AW \& Nielsen DR (1980). Spatial variability of soil physical properties in the field. In: HILLEL, D. (Ed.). Applications of soil physics. New York: Academic Press, 344p.

Zanão Júnior LA, Lana RMQ \& Guimarães EC (2007). Variabilidade espacial do $\mathrm{pH}$, teores de matéria orgânica e micronutrientes em profundidade em um Latossolo Vermelho sob semeadura direta. Ciência Rural, 37:1000-1007. 